\title{
Approche méthodologique de l'organisation des assolements afin de préserver la qualité des eaux souterraines
}

A methodological approach to crop rotation for the protective management of water resources

Methodische Untersuchung von Landnützungssytemen für das Management des Wasserschutzes

Marc Benoit et Céline Claude

\section{(2) OpenEdition}

\section{Journals}

Édition électronique

URL : http://journals.openedition.org/rge/2589

DOI : $10.4000 /$ rge.2589

ISSN : 2108-6478

Éditeur

Association des géographes de l'Est

Édition imprimée

Date de publication : 1 juin 2002

ISSN : 0035-3213

\section{Référence électronique}

Marc Benoit et Céline Claude, «Approche méthodologique de l'organisation des assolements afin de préserver la qualité des eaux souterraines », Revue Géographique de l'Est [En ligne], vol. 42 / 3 | 2002, mis en ligne le 14 décembre 2010, consulté le 08 septembre 2020. URL : http://

journals.openedition.org/rge/2589; DOI : https://doi.org/10.4000/rge.2589

Ce document a été généré automatiquement le 8 septembre 2020

Tous droits réservés 


\title{
Approche méthodologique de l'organisation des assolements afin de préserver la qualité des eaux souterraines
}

\author{
A methodological approach to crop rotation for the protective management of \\ water resources \\ Methodische Untersuchung von Landnützungssytemen für das Management des \\ Wasserschutzes
}

Marc Benoit et Céline Claude

\section{Introduction}

1 L'utilisation du territoire a toujours été un enjeu pour la société et les relations avec l'agriculture posent des questions qui sont devenues de plus en plus complexes. En effet, dans la plupart des situations étudiées par la recherche agronomique durant ces quarante dernières années, la recherche d'une meilleure productivité a été la question centrale de l'évolution des agricultures (Boulaine, 1992 ; 1998). Depuis quelques années, l'attention est portée sur les nouvelles fonctions de l'agriculture et des espaces ruraux (Allaire et al., 1996). La question du renouvellement des ressources naturelles présentes sur le territoire agricole devient prédominante (Leigh \& Johnston, 1994).

Or les agriculteurs, par les ajustements permanents de leurs pratiques productives, construisent l'utilisation du territoire agricole (Mannion, 1995). L'impact des activités agricoles sur le territoire est même un critère de définition des systèmes agraires dans une perspective historique (Grigg, 1974). L'évolution de l'utilisation des territoires agricoles dépend des acteurs en présence et de leurs choix d'activités. La gestion de ces territoires agricoles est donc un enjeu qui nécessite la prise en compte de la diversité 
des activités, et la maîtrise des processus bio-physiques de renouvellement des ressources.

3 Nous illustrons la démarche au travers de deux situations contrastées de territoires d'openfields, l'une, en Lorraine, où l'agriculture de polyculture-élevage est confrontée au problème de la protection de la qualité des eaux souterraines (Deffontaines et al., 1993), l'autre, dans la Marne où l'agriculture intensive doit faire face à la pérennisation d'une ressource en eau issue d'un bassin versant, celui de la Vesle.

Dans ces deux situations, nous sommes en présence d'évolutions d'openfields qui, par l'absence de haies et de talus, relient directement des qualités d'eau parcellaires à celles issues des bassins d'alimentation.

\section{Problématique}

\section{Position du problème, entre agronomie et géographie}

5 Nous centrons nos recherches sur les relations entre l'organisation des activités agricoles et les évolutions de ressources en eau autour d'un concept central: l'assolement, vu comme un indicateur de l'organisation de l'espace agricole et comme un révélateur des dynamiques territoriales.

6 Notre proposition s'inscrit dans l'axe des recherches-systèmes en agriculture (Sébillotte, 1996) qui repose sur trois principes méthodologiques: (i) partir de situations observées par enquête en exploitations agricoles, (ii) construire des modèles à partir d'études de cas, en utilisant les typologies d'exploitations comme moyen de prise en compte de la diversité, et (iii) élaborer, en partenariat avec les acteurs concernés, des outils de compréhension et d'aide à la gestion des phénomènes spatiaux (Christophe et al., 1996). Cette proposition est proche d'une approche agronomique anglo-saxonne et de travaux de géographes français. Les approches anglo-saxonnes se sont développées dans deux directions très différentes: l'une a étudié les acteurs en situation et les groupes locaux d'action, l'autre a proposé des modélisations de l'évolution de l'utilisation du territoire par des statistiques englobantes. Nous nous inspirons de la première, car nous pensons que les éléments de réponses doivent être trouvés au niveau où se posent les questions, c'est-à-dire souvent à un niveau local. La maitrise de l'utilisation du territoire se fait à un niveau intermédiaire entre les actes techniques des agriculteurs sur les parcelles et les régulations macro-économiques. Une école de recherche basée sur des modélisations multi-échelles de l'utilisation du territoire aborde cette question (Veldkamp et Fresco, 1997 ; de Koning et al., 1999). Certains modèles tentent de rendre compte de l'organisation territoriale de cette utilisation du territoire (Verburg et al., 1999). Ces travaux se sont multipliés depuis la prise en compte d'enjeux environnementaux par l'agriculture, lors de recherches visant à établir les bases de la durabilité de l'agriculture (Zander et Kächele, 1999). D'autre part, le développement de recherches sur l'agriculture de précision ${ }^{1}$ prend en compte l'organisation des assolements dans la mise en œuvre de ces nouvelles techniques et sur leurs effets sur le paysage (Gerber et al., 1998).

7 Nous retenons de ces travaux d'agronomes la nécessité de rendre compte de l'inscription spatiale de ces activités. Or, la façon dont les agriculteurs mettent en œuvre leurs productions se marque dans le territoire par la répartition des cultures. L'étude de cette organisation spatiale des systèmes de culture est l'une des tâches de 
l'agronome définie par M. Sebillotte (1974). Elargie à l'ensemble des productions végétales, y compris les cultures pérennes, la notion d'assolement en tant que répartition spatiale des cultures a du sens par rapport au fonctionnement des exploitations agricoles, mais aussi à l'interaction entre les activités agricoles et les autres activités du territoire. De plus, la répartition des cultures concerne différents acteurs: l'agriculteur met en valeur l'espace, le propriétaire définit les conditions d'utilisation de son patrimoine, les prescripteurs imposent des contraintes d'application des techniques, la société locale fait pression pour satisfaire un intérêt collectif. Cette attention aux enjeux territoriaux de l'activité agricole sensibilise plus encore que par le passé les agronomes aux concepts et démarches de géographes (Benoît et Papy, 1997).

8 L'approche géographique de l'environnement est à la fois naturaliste et sociale, c'est-àdire qu'elle s'intéresse aux rapports entre nature et société. Le titre de l'ouvrage dirigé par Y. Veyret en 1998 : L'érosion entre nature et société, est significatif de ces nouvelles approches à l'interface de la géographie physique et de la géographie humaine. Ces problématiques environnementales ont donc l'intérêt de rompre avec le dualisme de la géographie : l'ensemble des questions et des méthodes qu'elles induisent articulent les approches naturelles et sociales, et sont susceptibles de créer de nouveaux objets de recherche.

Il s'agit donc de prendre en compte la dynamique des composantes du milieu en y intégrant le rôle des facteurs humains. Il est possible d'évoquer une "géodynamique ", où les éléments physiques et biologiques interagissent selon des modalités qui varient avec les contextes humains, techniques et sociaux. Dans le cas d'une approche "hydrosystémique ", il faudra prendre en compte les facteurs (aussi bien physiques qu'humains) qui règlent la dynamique hydrologique et conditionnent la gestion de la ressource « eau » à différentes échelles spatiales et temporelles.

10 Ces nouvelles perspectives expliquent les multiples contacts que les géographes entretiennent désormais avec des chercheurs d'autres disciplines, puisqu'il s'agit pour les problématiques environnementales d'intégrer les diverses dynamiques dans des relations systémiques.

11 La démarche que nous suivrons ici s'inscrit dans un courant géographique qui se situe à l'interface de la géographie humaine et de la géographie physique, et examine les interrelations entre l'homme et l'environnement. Nous n'étudierons qu'une des ressources environnementales: l'eau, dans ses interrelations avec les pratiques agricoles organisées par l'homme.

12 L'assolement constitue une de ces pratiques à l'origine de phénomènes qui influencent les systèmes vivants et les hydrosystèmes qu'ils modifient.

\section{La question de la protection des ressources en eau}

13 Nous mettons à l'épreuve cette modélisation de l'organisation des assolements sur deux chantiers de recherche de l'INRA-SAD ${ }^{2}$ dont les questions initiales étaient très différentes. Il s'agit de montrer que l'assolement est un indicateur spatial de fonctionnement de ces systèmes agraires permettant de suivre l'évolution de l'utilisation des territoires concernés. Dans ces deux exemples, l'assolement est l'indicateur de l'organisation spatiale des cultures. Le fonctionnement et la dynamique des activités sont décrits par l'occupation du sol, les types de couverts ou de cultures, 
aux différents niveaux d'organisation concernés, la parcelle, l'exploitation, le bassin d'alimentation. Les Systèmes d'Information Géographique et l'analyse d'images de télédétection sont les outils d'analyse spatiale utilisés pour décrire et comparer ces assolements. Ils fournissent les représentations spatiales du fonctionnement et de l'évolution du système à présenter aux acteurs concernés.

14 L'équipe du SAD Versailles-Dijon-Mirecourt a été impliquée dans la gestion des ressources en eau et la prévention de la pollution par les nitrates, en particulier dans le bassin des eaux minérales de Vittel (Deffontaines et al., 1993). La qualité de l'eau dépendant de l'occupation du sol, la recherche a permis d'identifier les bassins «à risque " et les exploitations agricoles concernées. Gérer l'eau consiste alors à transformer les systèmes de culture pour qu'ils soient plus respectueux de l'environnement.

15 Les ressources en eau s'élaborent dans les bassins d'alimentation. Elles sont régies par les gestionnaires de l'eau (maires pour les régies communales, présidents des syndicats intercommunaux) et les gestionnaires du territoire des bassins d'alimentation (agriculteurs, forestiers, résidents) (Salou, 1992). Chaque parcelle culturale sur laquelle un système de culture est mis en œuvre participe à deux entités spatiales fonctionnelles: un bassin d'alimentation et une exploitation agricole. On peut avoir deux lectures des territoires de l'eau. D'une part la reconnaissance des occupations du sol d'un bassin d'alimentation permet d'identifier le risque qu'elles font courir à la qualité de l'eau selon la conduite agronomique des couverts végétaux. Cette lecture est utile pour établir un diagnostic préalable à toute action préventive (Benoit, 1992 ; Benoît et al., 1995). L'assolement est l'indicateur de risque. D'autre part, la structure du bassin permet de définir le taux d'implication de chaque exploitation et ainsi, de cibler les interventions. Cette compréhension s'avère nécessaire quand des changements de pratiques doivent être envisagés en négociation entre les gestionnaires de l'eau et ceux du territoire. L'assolement est l'enjeu concret de la négociation, ainsi que le souligne Claude (2001) au sujet de l'ensemble du bassin de la Vesle.

16 Les caractéristiques du milieu physique, nappes phréatiques multicouches ou stratigraphie superposant des couches à perméabilité différenciée, peuvent retarder l'observation des conséquences des activités humaines sur les eaux des nappes phréatiques donc in fine dans l'eau des sources. Cependant, les structures géologiques ne peuvent induire des évolutions de qualités importantes une fois la qualité des eaux construite sous la zone racinaire.

\section{L'assolement comme indicateur de l'utilisation du territoire et de son évolution}

17 Nous formalisons l'assolement, répartition des cultures dans l'espace, comme une organisation de l'utilisation du territoire. C'est pour nous un bon indicateur du fonctionnement des exploitations agricoles; il a pour intérêt d'intégrer la dimension spatiale, qui est importante dans les questions de maîtrise des processus naturels. Il rend compte de la double signification du terme organisation, dans son acception active de mise en œuvre des activités et dans sa résultante, la répartition spatiale des couverts végétaux. Il présente un point de vue riche sur l'utilisation du territoire, car il ne s'enferme pas dans la seule vision agricole, mais a du sens pour les autres acteurs de 
l'espace rural. De plus, c'est un révélateur des dynamiques à l'œuvre; il fournit des éléments concrets de comparaison pour cerner les évolutions du territoire.

\section{a. L'utilisation du territoire se fait au travers d'actes techniques} territoire (Benoît, 1990). Ainsi, la production laitière bovine nécessite une répartition des pâturages de vaches laitières à proximité du siège de l'exploitation, à moins que l'on ne passe à la traite au parc, qui se marque par des blocs de pâtures plus éloignés, ou que l'on pratique l'affouragement en vert, à partir de parcelles cultivées. Nous avons montré que non seulement la répartition des cultures était un indicateur du fonctionnement des exploitations, mais que les caractéristiques spatiales des parcelles étaient diversement mobilisées pour permettre telle ou telle production (Benoît, 1985). Le territoire agricole est composé de parcelles aux caractéristiques multiples que l'on peut évaluer de l'extérieur et qui sont également l'objet de perceptions et représentations culturelles et affectives des utilisateurs (Morlon \& Benoît, 1990) : milieu naturel (sol, topographie, climat...), superficie et forme, distance et facilité d'accès depuis les bâtiments d'habitation et d'exploitation, appropriation et contraintes sociales à respecter, d'ordre contractuel, collectif, légal ou réglementaire. Les arrangements entre parcelles rendent compte de l'intégration de ces différents critères pour la mise en œuvre des activités. Les règles issues d'enquêtes en exploitations permettent de décrire ces situations et de les représenter spatialement (Le Ber \& Benoît, 1998).

\section{c. L'utilisation du territoire interfère avec les processus naturels} L'assolement fournit une indication de l'importan
parcelle par rapport aux logiques agronomiques.

Si les parcelles agricoles sont appropriées par différents acteurs et entrent dans des logiques de fonctionnement humain, elles sont aussi localisées dans un milieu et subissent des processus naturels. Ainsi, une même parcelle, avec les mêmes potentialités agronomiques, pourra prendre une valeur totalement différente dans une 
gestion agricole, selon qu'elle est ou non soumise à une forte influence de processus bio-physiques. Par exemple les phénomènes d'érosion ou de ruissellement s'appliquent le long de ravines en entraînant une dépréciation du terrain, pas obligatoirement en suivant les limites des parcelles de propriété (Martin et al., 1998). Pour rendre compte de ces discontinuités dans l'espace, provoquées par des phénomènes d'ordre biophysique, il faut se donner les moyens d'analyser l'espace dans sa dimension continue (Benoît et al., 1997). C'est le cas dans tous les problèmes d'environnement.

\section{d. L'utilisation du territoire nécessite des arbitrages entre individuel et collectif}

24 L'assolement fournit une indication du poids des contraintes collectives par rapport aux logiques individuelles.

Les parcelles agricoles appartiennent à des exploitations et doivent donc répondre, dans leur utilisation, à la logique de fonctionnement des unités de production individuelles, mais elles sont aussi incluses dans des logiques collectives, au niveau des villages par exemple. Cela conduit à une organisation en sole, alors qu'une organisation concentrique rendrait mieux compte, par exemple, des contraintes de travail liées à la distance (Benoît, 1990). Il y a donc des arbitrages entre différentes logiques et les hiérarchies qui se dégagent se concrétisent dans les assolements réalisés.

\section{e. L'utilisation du territoire évolue dans le temps}

L'assolement permet de comparer les évolutions du territoire en référence à l'évolution du contexte technique et socio-économique.

Les caractéristiques spatiales sont perçues en termes de contraintes ou atouts, qui se modifient dans le temps en fonction des objectifs de production, des techniques d'aménagement (drainage, irrigation, bocages, remembrement, ...), des moyens physiques et humains de travail sur les parcelles et de transport, des règles de conduites liées à la protection de l'environnement physique (bassin d'alimentation en eau potable à protéger, cultures en courbes de niveau pour freiner l'érosion), des rapports de prix, des réseaux de commercialisation, des mesures réglementaires et des relations entre groupes sociaux. L'analyse de l'évolution des assolements révèle ces changements.

\section{f. L'utilisation du territoire met en jeu plusieurs niveaux d'organisation}

28 L'assolement a du sens pour chacun des niveaux d'organisation à considérer. Plus encore, il peut être un fil directeur, pour passer d'un niveau à l'autre.

L'utilisation du territoire doit être considérée à différents niveaux d'organisation, combinaison d'échelles de temps et d'espace (Thenail, 1996). C'est l'échelle de la campagne annuelle qui détermine la localisation de certaines cultures, mais c'est la stratégie à plus long terme qui commande les mises en place d'aménagements et la différenciation des blocs de culture. Ce n'est pas seulement l'échelle du territoire de l'exploitation, mais aussi celle du finage villageois et de la petite région qu'il faut considérer pour tenir compte des effets d'appropriation et d'appartenance. Il existe aussi des niveaux intermédiaires entre les intérêts individuels et collectifs, entre le naturel et l'humain, entre l'action et la perception, dont nous allons détailler l'exemple des relations assolement - qualité des eaux. 


\section{Les données prises en compte et les méthodes d'acquisition :}

\section{Un modèle empirique d'explication des évolutions de qualités des eaux}

La variable à expliquer est ici la qualité des eaux. Celle-ci résulte du fonctionnement hydrique et hydrogéologique d'un bassin d'alimentation et des activités qu'il supporte. Pour chaque bassin, la qualité moyenne annuelle est la moyenne des concentrations nitriques mesurées successivement, chaque mesure étant pondérée par le débit instantané de chaque source. D'autre part, nous formulons l'hypothèse que cette qualité d'eau est le résultat des pertes nitriques des diverses occupations du sol qui composent chaque bassin. L'hypothèse formulée alors est la suivante : la qualité ([NO3]) de l'eau issue d'un bassin d'alimentation est la moyenne, pondérée par leur surface, des qualités de l'eau provenant des diverses occupations du sol couvrant le bassin. La teneur en nitrates ainsi calculée pour chaque source s'exprime selon la formule suivante :

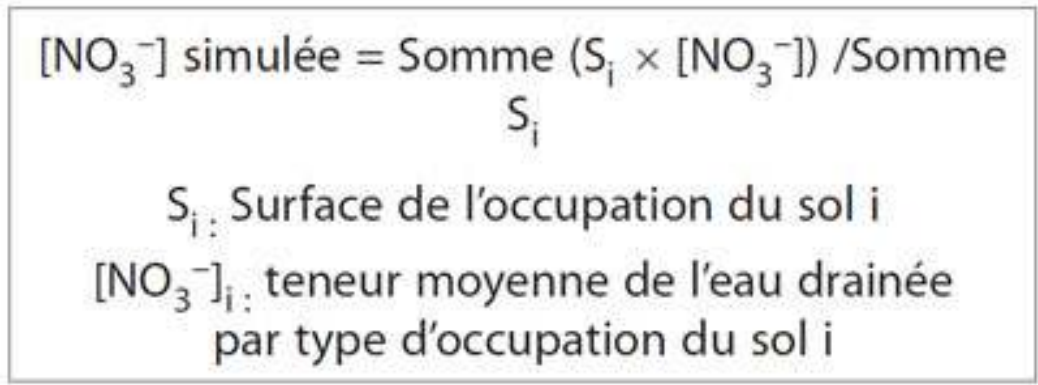

31 Les effets du climat, ceux des pratiques agricoles et les types de sols sont intégrés dans

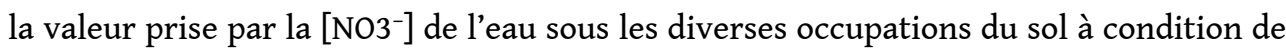
disposer de mesures réparties au cours du temps comme nous l'expliquerons au paragraphe suivant. Le changement de l'assolement du bassin est mesuré à travers la mesure des surfaces des diverses occupations du sol.

Les différents types d'occupation des sols agricoles, ainsi que les teneurs moyennes en nitrates en fonction de la nature du couvert végétal, apparaissent comme les variables majeures dans l'explication de la qualité des eaux souterraines. Il apparait donc important de développer des méthodes permettant de suivre au mieux ces surfaces et de modéliser les causes de ces changements. L'objectif a été, et demeure de restaurer la qualité des eaux en modifiant les assolements sur les bassins d'alimentation concernés.

\section{Les outils de ce modèle empirique}

Abordons maintenant la détermination spatio-temporelle des deux variables principales expliquant la qualité des eaux en région d'openfield : l'assolement et la qualité des eaux par sole. 


\section{a. Evaluation de l'assolement des bassins d'alimentation}

34 La mesure de la surface des cultures, prairies permanentes et forêts, est réalisée soit par observation directe, soit par enquêtes auprès des agriculteurs exploitant le bassin concerné, soit en utilisant les bases statistiques de TerrUti ${ }^{3}$.

La mesure par observation s'effectue par des suivis de terrain au cours desquels, par observation visuelle directe, sont notés en début juin, les couverts végétaux de la campagne agricole. Cette méthode nécessite environ deux journées de terrain par millier d'hectares à cartographier. L'enquête agricole consiste à interroger les agriculteurs sur l'utilisation de leurs parcelles à partir d'une photographie aérienne sur laquelle ils dessinent leurs parcellaires et en décrivent ensuite l'utilisation successive des parcelles (Heydel et al., 1997). La digitalisation de ces données sous un Système d'Information Géographique permet d'obtenir des états et des évolutions de l'utilisation du territoire.

36 Nous avons étendu ces démarches à des échelles micro-régionales, celles des petites régions agricoles. Ainsi, nous rendons compte de l'évolution d'assolements au cours du temps par l'ensemble des successions culturales. Les données initiales proviennent des enquêtes annuelles Terruti que nous traitons à l'aide de Chaînes de Markov ${ }^{4}$. Les traitements obtenus donnent des liens représentant les successions culturales, et à une date donnée, l'épaisseur de ces liens fournit l'assolement annuel.

\section{b. Evaluation des lixiviations nitriques par occupation du sol}

La deuxième variable à instruire est la qualité de l'eau par système de culture. Pour ce faire, nous avons opté pour un suivi continu des pertes nitriques à l'échelle d'un échantillon de parcelles d'agriculteurs (Benoît et al, 1995), par implantation de sites à bougies poreuses enterrées à 1,1 mètre de profondeur.

Depuis 1989, 52 parcelles sont suivies en continu dans diverses situations lorraines (périmètre de Vittel, opération Ferti-Mieux du Haut-Saintois à cheval entre la Meurtheet-Moselle et les Vosges, Unité Expérimentale SAD à Mirecourt). Les prélèvements ont lieu tous les 14 jours et après les plus importants épisodes pluvieux (supérieurs à 40 $\mathrm{mm})$.

Donc, pour chaque parcelle et pour chaque campagne culturale, nous disposons de 17 à 29 dates de mesures par an, qui prennent en compte les aléas climatiques et en particulier les périodes pluvieuses. Suivant les années, ce chiffre varie car il est lié aux périodes de sécheresse pendant lesquelles les bougies poreuses sont «taries». Cette forte fréquence de mesure au cours du temps nous permet de prendre en compte les successions d'états créées sur la parcelle par la séquence des techniques culturales appliquées. En effet, qu'il s'agisse de travaux du sol, de fertilisations organiques ou minérales, d'implantation de cultures intercalaires pièges à nitrate, nous échantillonnons à un pas de temps suffisamment fin pour prendre en compte les effets sur les pertes en nitrate induits par telle ou telle pratique. L'intérêt majeur de ce suivi est la prise en compte des aléas temporels (relatifs au climat et aux pratiques agricoles) enregistrés sur les parcelles agricoles.

Pour chacune de ces dates et chaque site parcellaire, nous disposons de 7 prélèvements car nous avons implanté 7 bougies par site. Ainsi, une mesure moyenne annuelle par parcelle est l'intégration de 7 mesures par date sur une vingtaine de dates au cours de la 
campagne (Benoît et al, 1995). Chaque mesure calculée par culture pour une année est donc statistiquement robuste car basée sur environ 150 mesures individuelles (une bougie à une date). Ainsi, les occupations de sol « colza » sont évaluées sur 8 « parcelles-années " (8 parcelles ayant porté du colza une année pendant la période d'observation) soit un ensemble de 749 mesures individuelles dans ce cas précis. Pour conclure, les implantations de ces sites à bougies poreuses ont été réalisées grâce à la collaboration d'agriculteurs lorrains, offrant ainsi la possibilité d'une observation in situ et en continu de leurs pratiques agricoles (assolements et itinéraires techniques) et de leurs effets sur la qualité de l'eau.

\section{Résultats}

\section{Evaluation d'une qualité d'eau à différentes échelles par l'étude de l'évolution de l'utilisation du territoire agricole}

41 Les deux paramètres à prendre en compte sont donc: les occupations de sol et les teneurs en nitrate par occupation de sol.

En partant de la liste annuelle des occupations de sol enquêtée par le dispositif TerrUti, nous pouvons en décliner deux interprétations: l'assolement annuel en sommant les occupations de sol de chaque culture et prairie, l'évolution temporelle des cultures en suivant les successions culturales (tableau 1, fig. 1 et fig. 2).

Tableau 1 : Evolution de l'assolement en Champagne crayeuse (en \%)

\begin{tabular}{|l|c|c|c|c|c|c|c|}
\hline & 1992 & 1993 & 1994 & 1995 & 1996 & 1997 & 1998 \\
\hline Blé & 31,4 & 30,3 & 31,7 & 31,6 & 32,7 & 31,8 & 32,6 \\
Pois & 13,9 & 13,0 & 10,7 & 10,3 & 9,7 & 10,1 & 10,3 \\
Orge & 13,8 & 11,6 & 11,6 & 12,3 & 15,8 & 16,3 & 17,2 \\
Luzerne & 12,2 & 11,1 & 10,1 & 9,6 & 9,3 & 9,9 & 10,1 \\
Betteraves & 12,1 & 11,6 & 12,9 & 13,2 & 11,9 & 13,4 & 11,8 \\
Colza & 3,0 & 2,0 & 3,3 & 4,5 & 4,1 & 3,4 & 4,1 \\
Maïs & 2,9 & 3,0 & 1,7 & 1,9 & 2,4 & 2,6 & 2,3 \\
Pomme de terre & 2,5 & 2,5 & 2,4 & 2,9 & 2,5 & 3,0 & 2,7 \\
Tournesol & 2,4 & 1,3 & 1,9 & 2,0 & 1,7 & 1,5 & 1,0 \\
Prairies permanentes & 1,9 & 1,8 & 1,8 & 1,8 & 1,8 & 1,8 & 1,8 \\
Jachères & 0,4 & 7,4 & 7,7 & 5,6 & 3,5 & 1,7 & 1,4 \\
\hline
\end{tabular}

Sur l'exemple du bassin de la Vesle en Champagne crayeuse nous avons ainsi procédé à la mise en évidence de l'évolution faible de l'assolement de 1992 à 1998.

La figure 1 illustre clairement cette stabilité, à l'exception d'une augmentation des surfaces en orge et en colza, et une forte diminution des surfaces en jachères. La figure 2 montre que l'augmentation des surfaces en orge a eu lieu de 1994 à 1997, celle des surfaces en colza s'étale de 1993 à 1995, enfin la diminution de la jachère est régulière de 1995 à 1997. 
Figure 1 : Assolements de Champagne crayeuse (en 1992 et 1998)

Evolution des assolements en Champagne Crayeuse entre 1992 et 1998

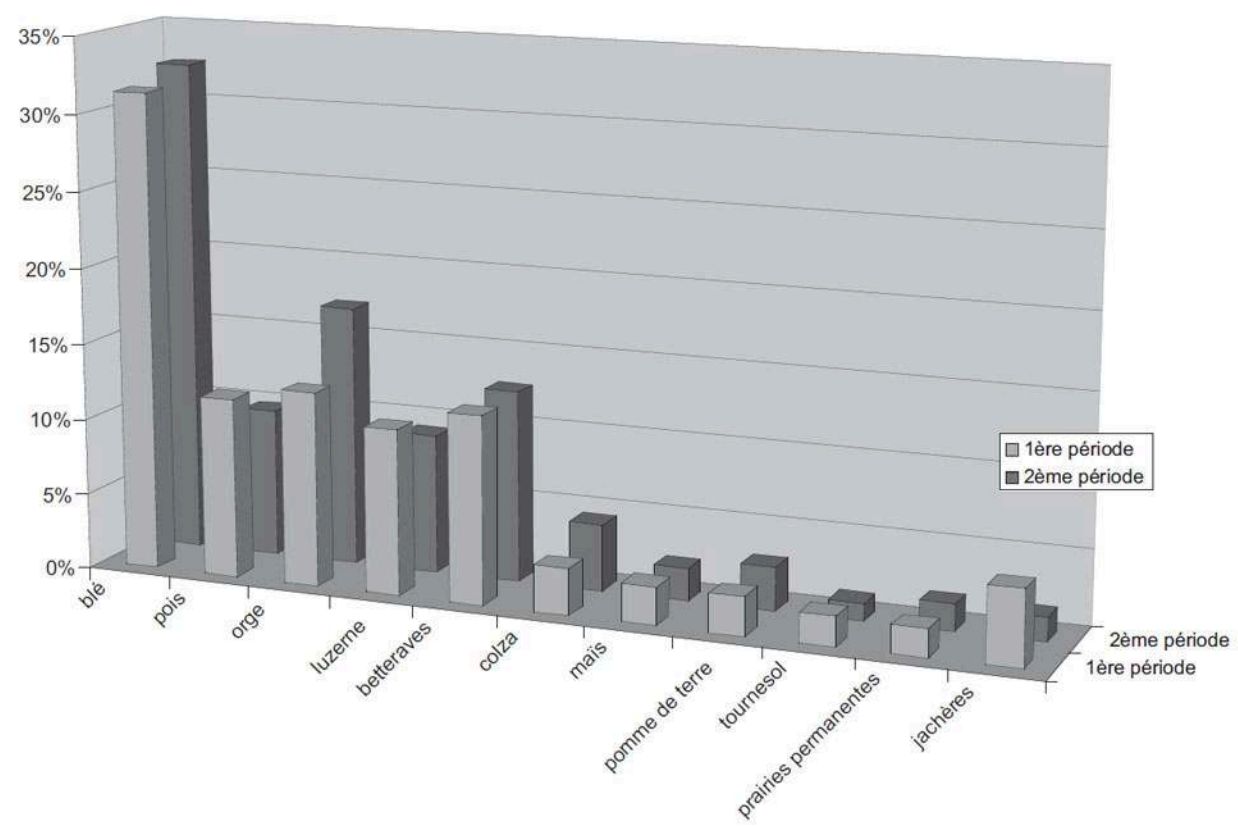

Figure 2 : Evolution l'assolement de 1992 à 1998 en Champagne crayeuse
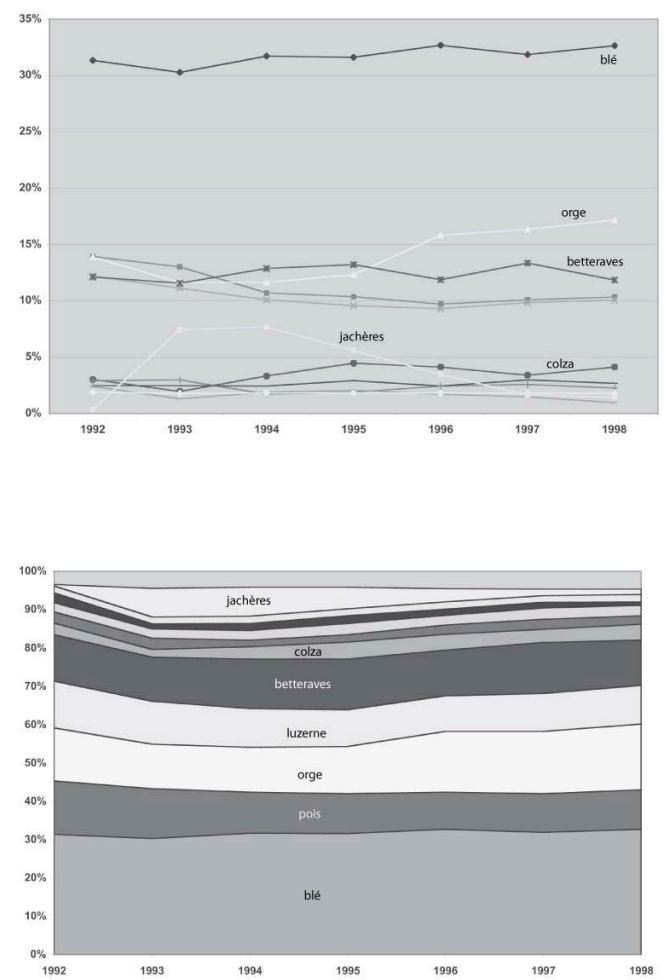

Une autre lecture de ces assolements est de rendre compte des dynamiques des successions culturales. En effet, ces deux points de vue sur le territoire sont liés: chaque année un agriculteur se fixe une surface à cultiver pour chacune des cultures 
ou prairie qu'il a choisie, mais il doit tenir compte pour chaque parcelle de la culture présente l'année précédente, car chaque culture ne suit pas aléatoirement une autre. Par exemple, les couples colza-blé sont fréquents mais à l'inverse les couples colzabetteraves sont inexistants.

Pour en rendre compte, nous avons élaboré avec des statisticiens une procédure de dépouillement de ces données qui rende compte sur un même graphique des successions de culture et de l'importance des différentes cultures une année donnée (Benoît, Le Ber, Mari, 2001). La figure 3 illustre cette méthode.

Figure 3 : Schéma explicatif des successions culturales traitées par «CarrotAge » à partir des données TerrUti

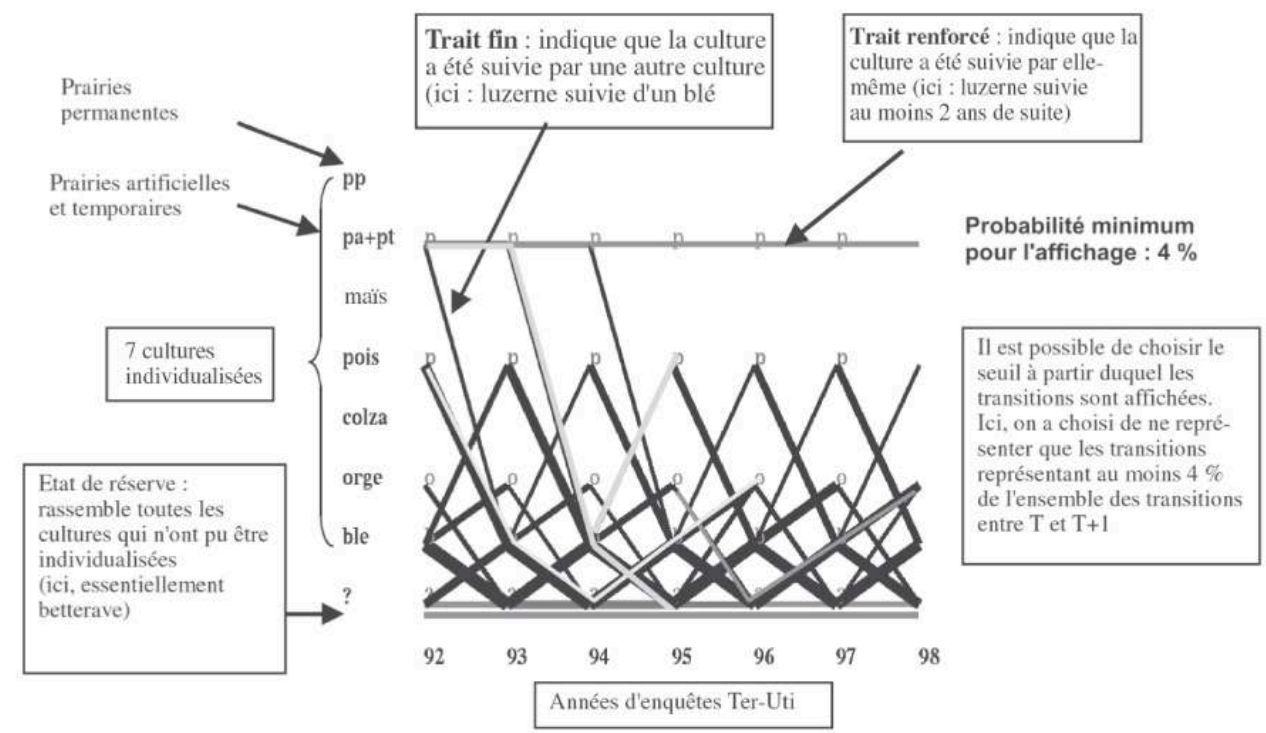

Pour lire un diagramme de Markov et retrouver les principales successions, on peut suivre les traits de transitions les plus épais et retrouver ainsi une succession sur 5 ans du type : Pois-Blé-Betterave-BléOrge, ou des successions en 3 ans du type Betterave-Blé-Orge. Il est par contre difficile de dire quelles seront les cultures implantées de préférence après 2 ans de luzerne une année de blé.

La stabilité que nous avons constatée sur les assolements se retrouve également dans les successions culturales (figure 4). 
Figure 4 : L'évolution régionale des successions culturales

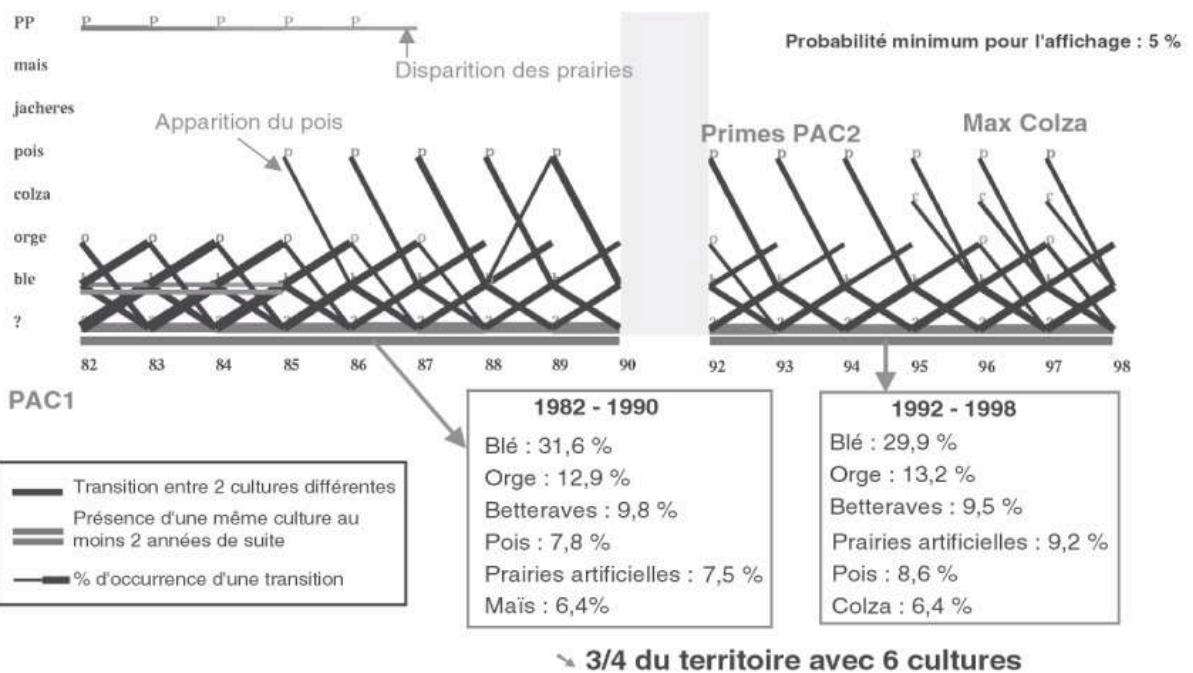

Recherche de régularités dans l'utilisation des terres agricoles : diagramme et listing « 1 culture » sur le département de la Marne.

Dans le cas du Plateau lorrain sud, le graphique fait ressortir un ensemble de prairies en monoculture qui régressent, et une succession dominante à base de colza, blé et orge qui progresse (figure 5).

Figure 5 : évolution des successions culturales dans la Petite Région Agricole Plateau lorrain (PP = prairies permanentes)

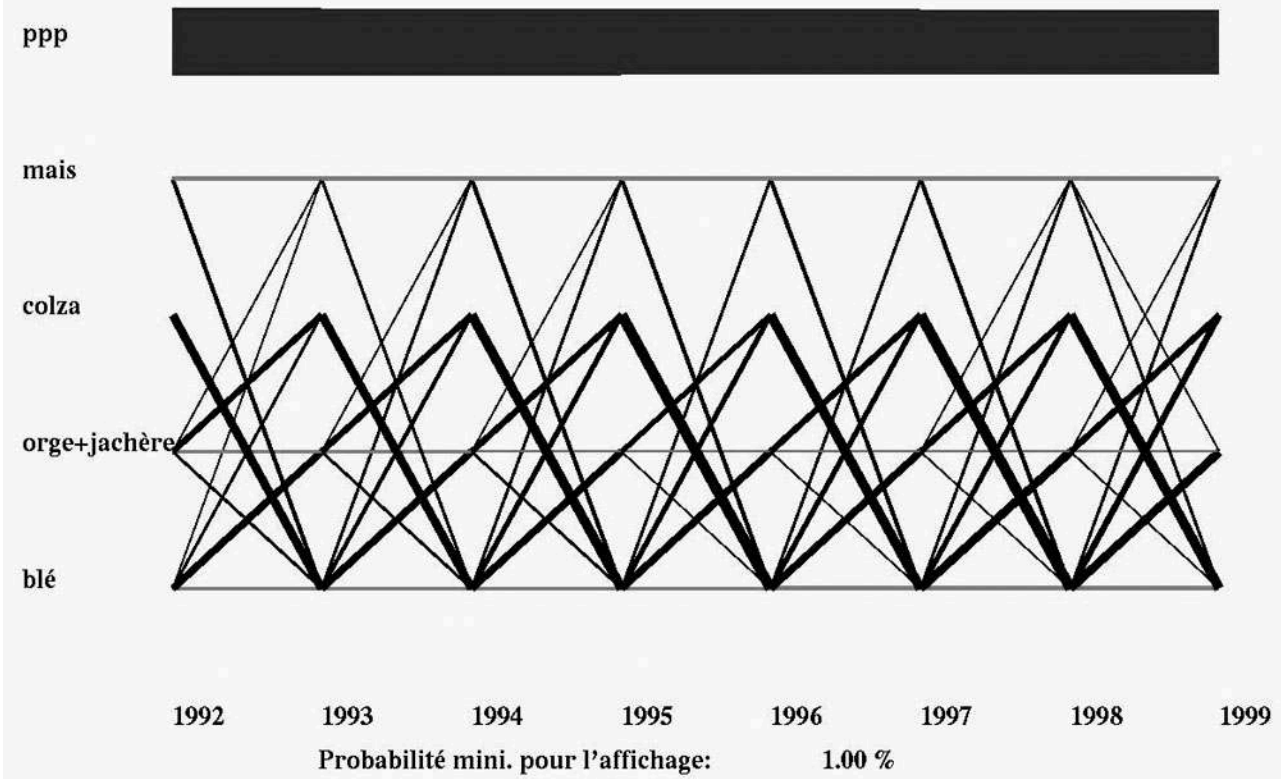

Les monocultures sont représentées par les traits horizontaux, les successions par des traits obliques entre les différents couverts.

A titre d'exemple, voici les résultats issus d'une situation du début des années 1990 du sud Plateau lorrain (tableau 2). 
Tableau 2 : nombre et valeurs des mesures de lixiviations des nitrates, réalisées de 1989 à 1992 , sur les principaux couverts végétaux en Plateau Lorrain (Gaury, 1992)

\begin{tabular}{|l|c|c|c|}
\hline \multicolumn{1}{|c|}{$\begin{array}{c}\text { Couverts végétaux } \\
\text { effectuées }\end{array}$} & Nombre de mesures & $\begin{array}{c}\text { Moyenne } \\
\text { (mg NO3/l) }\end{array}$ & Ecart-type \\
\hline Forêts & 5 & 2 & - \\
Prés de fauche & 9 & 19 & 14 \\
Pâtures & 18 & 31 & 25 \\
Prairies temporaires & 3 & 28 & - \\
Luzernes & 13 & 23 & 25 \\
Blé d'hiver & 27 & 46 & 25 \\
Orge d'hiver & 27 & 46 & 52 \\
Colza & 8 & 120 & 20 \\
Céréales de printemps & 8 & 32 & 77 \\
Maïs fourrager & 28 & 126 & \\
\hline
\end{tabular}

\section{Intérêt de l'itinéraire méthodologique pour les cas étudiés}

L'assolement est considéré comme un produit du fonctionnement des activités agricoles en paysages d'openfields et comme un indicateur de leurs transformations. Sa représentation est aussi un moyen de communication et de négociation entre acteurs. Nous illustrons les étapes de l'itinéraire méthodologique à partir des deux exemples étudiés avant de présenter les possibilités de généralisation de la démarche.

51 Nous avons identifié cinq étapes dans l'itinéraire méthodologique que nous avons mené pour le diagnostic de la protection des eaux. Elles sont issues de la comparaison de nos deux analyses, où nous avons identifié les points de passage obligés, les différences et les analogies.

\section{Etape 1 : identifier les niveaux d'organisation}

Le choix des niveaux d'organisation à considérer est déterminant (Muxart et al., 1992). Ils constituent le référentiel que l'on se donne pour la modélisation, c'est-à-dire à la fois le cadre du système étudié, mais aussi l'unité d'observation. Certaines entités spatiales sont facilement identifiées, ce sont les unités élémentaires d'observation et l'ensemble de la zone d'étude : dans notre cas, le bassin d'alimentation considéré. Ceci même si le travail des hydrogéologues pour les déterminer est parfois délicat, en particulier dans des régions à soubassement karstique. Elles constituent bien souvent les données de départ. Dans les deux cas étudiés, ce sont les parcelles qui sont les unités élémentaires retenues, considérées comme stables sur un pas de temps de quelques années. Pour ce qui est de la question de la préservation de la qualité des ressources en eau, tant dans la Vesle qu'en Lorraine, c'est le bassin d'alimentation de chaque ressource en eau. Le processus visé, la préservation des ressources en eau, construit le niveau englobant de notre approche (Canter, 1997). La période de référence est de l'ordre de la décennie, car il faut considérer l'histoire de la construction de ces zones comme entités de décision et d'action.

Mais quels sont les niveaux intermédiaires qui ont du sens par rapport aux questions posées ? Nous intéressant à la façon dont les agriculteurs peuvent gérer le territoire par leurs activités, nous choisissons de considérer les exploitations agricoles dans leur fonctionnement technique (Osty, 1994 ; 1996). En effet, pour mener à bien l'analyse, il 
faut se placer au niveau où on peut agir et avoir une certaine connaissance des logiques de fonctionnement.

\section{Etape 2 : formaliser des indicateurs spatiaux de fonctionnement} avons retenus. En Lorraine, les occupations du sol ont pu être reliées au risque de pollution par les nitrates (Benoît et al., 1995). Dans les bassins d'alimentation, les dynamiques des assolements résultent du fonctionnement des exploitations agricoles et de leur emprise foncière et déterminent les risques vis-à-vis des ressources en eau (Benoît et al., 1997). Dans le bassin de la Vesle, notre première image de recherche est bâtie sur la même hypothèse (Claude, 2001).

Ainsi, il s'agit de construire, à partir de la connaissance que l'on a des fonctionnements et des mécanismes, des indicateurs spatiaux qui soient repérables avec les outils d'observation, par exemple, la télédétection (Girard et al., 1990). En Lorraine, l'évaluation des surfaces de cultures, prairies permanentes et forêts s'est faite en combinant trois méthodes (Gaury, 1992) qui sont: (i) l'interview direct de tous les agriculteurs intervenant sur le périmètre du bassin versant pour connaître la structure de leurs exploitations, (ii) la photo-interprétation pour extrapoler, à partir d'occupations du sol connues, les règles de reconnaissance des couverts sur la couleur, la texture et les ombrages de la photographie, (iii) les observations de terrain par notation visuelle, début juin, des couverts végétaux de la campagne agricole. Nous pensons que le traitement d'images satellitaires (Girard \& Benoît, 1990) serait un outil particulièrement bien adapté à cette question de recherche si nous voulons travailler sur de grands bassins versants. La numérisation de ces données dans un système d'information géographique permet de représenter les états et les évolutions de l'utilisation du territoire (Benoît et al., 1997). Dans le bassin de la Vesle, nous avons privilégié l'étude des séries chronologiques des enquêtes Terr-Uti (Benoît et al., 2001) pour rendre compte de l'évolution des assolements et des dynamiques des successions culturales ${ }^{5}$ (Claude, 2001).

\section{Etape 3 : mettre en correspondance mode de fonctionnement et structure spatiale}

Il s'agit maintenant de construire un modèle assurant la correspondance entre le mode de fonctionnement de l'exploitation agricole qui détermine la logique d'organisation des assolements et la structure spatiale ainsi créée, représentée par l'assolement observé. Il nous faut aussi valider la relation inverse : l'assolement, indicateur spatial mesurable par différentes techniques d'observation, doit être également révélateur de la façon dont les exploitants organisent leur espace, pour être discriminant des différents types d'exploitation.

Dans les deux cas étudiés en Lorraine et en Champagne, le modèle construit est relativement simple car il ne différencie pas les exploitations. Pour évaluer la qualité de l'eau, nous avons privilégié cette méthode nécessitant la connaissance de l'assolement des bassins et le suivi de la solution du sol par bougies poreuses sur des sites permanents, avec prélèvements d'eau bimensuels (Ballif \& Muller, 1990) A partir du référentiel parcellaire précédemment établi qui fournit un taux de nitrate pour chaque occupation du sol, et connaissant de manière exhaustive l'utilisation du territoire une année donnée, il est possible de simuler, à partir du modèle, les teneurs en nitrate et de les comparer aux valeurs mesurées. C'est ce qui a été fait pour un petit bassin versant 
de 984 ha, en plateau lorrain, celui du petit Vair, de 1975 à 1991 : les écarts entre teneurs simulées et teneurs mesurées sont toujours inférieures à $4 \mathrm{mg} / \mathrm{l}$ de [NO3-] (à comparer à la valeur de $125 \mathrm{mg} / \mathrm{l}$ pour le maïs fourrager) (Benoît et al, 1995). On peut dire que ce modèle, simple, est suffisant pour rendre compte des risques de pollution dans le périmètre de protection (Mary et al., 1997).

\section{Etape 4 : étendre spatialement cette relation} d'alimentation, pour l'évaluation du risque de pollution. Le diagnostic s'appuie donc sur les évaluations faites. Le niveau des exploitations agricoles n'intervient que dans la mesure où l'on veut rechercher les exploitations impliquées dans ce risque, ce que l'on fait par le calcul du taux d'implication' (Benoît \& Papy, 1997). Pour la protection des ressources en eaux, les modalités techniques et organisationnelles des changements de systèmes de culture font intervenir de nouveaux niveaux d'organisation, tels que les groupes d'agriculteurs voisins ou les CUMA d'organisation du travail, qu'il serait intéressant d'étudier.

\section{Conclusion}

La modélisation des relations existant entre les systèmes techniques et le territoire doit produire des indicateurs spatiaux de fonctionnement, utilisables par les acteurs concernés par la gestion des territoires. Nous prenons l'assolement comme indicateur spatial de fonctionnement. Ce concept est opératoire pour les agronomes, car il est constitutif de leur discipline et central dans les enjeux du fonctionnement des exploitations agricoles dans leurs relations avec l'environnement. Il est intéressant 
pour les géographes car il constitue un descripteur facilement identifiable dans le paysage agricole des territoires d'openfields.

Cette proposition amène un renouvellement de la réflexion sur les systèmes de culture en agronomie, qui ont jusqu'alors porté leur attention surtout sur les aspects temporels et stratégiques. La prise en compte de la dimension spatiale et organisationnelle amène à interroger le modèle d'action des agriculteurs pour s'ajuster aux situations locales et aux événements conjoncturels, telles les évolutions de la PAC, qui conditionnent les choix de certaines cultures. Elle permet en particulier d'améliorer l'appréhension de la situation par les acteurs qui doivent résoudre le problème de la gestion des assolements (Maxime et al., 1995). Elle fournit également des ouvertures nécessaires sur l'environnement de l'exploitation, qui déterminent la capacité des systèmes techniques de production à s'adapter aux nouveaux contextes (Osty et al., 1998). Enfin, elle place délibérément l'objectif de la recherche dans le champ de l'action, en ne s'éloignant pas des objets concrets manipulés par les acteurs.

Dans le champ de la modélisation, notre proposition s'inscrit dans le courant de la méthode des modèles (Legay, 1986). Elle reprend les grandes étapes d'une démarche de modélisation proposée par Legay (1997) : analyse de situation, position du problème, choix d'un point de vue et expression des hypothèses, formalisation du modèle, paramétrage et confrontation des résultats aux données de l'expérience. Elle insiste sur l'organisation, comme propriété des systèmes complexes, au cœur de la modélisation systémique (Le Moigne, 1990). Enfin, du fait de son entrée spatiale, elle bénéficie des avancées récentes de la géographie qui s'occupe des lois d'organisation de l'espace, de l'occupation et de l'utilisation des territoires (Brunet, 1997). L'agronomie y trouve une possibilité de renouvellement (Deffontaines, 1998 ; Benoît et al., 1998) en s'alliant à la géographie dans ses chantiers de recherche.

\section{BIBLIOGRAPHIE}

Nous remercions les DRAF de Lorraine et de Champagne-Ardennes pour leur fourniture des données TerrUti et Céline Schott (INRA SAD Mirecourt) pour son aide aux traitements de ces données sur la Champagne crayeuse.

Allaire G., Hubert B., LANGlet A. (eds) (1996). - Nouvelles fonctions de l'agriculture et de l'espace rural. Enjeux et défis identifiés par la recherche. Actes Colloque Toulouse, 17-18 décembre 1996. Paris, INRA, $313 \mathrm{p}$.

BAlLIF J.L. \& Muller J.C. (1990). - Les bougies poreuses et les lysimètres. Perspectives Agricoles, 144, 72-81.

BENoîT M. (1985). - La gestion territoriale des activités agricoles. L'exploitation et le village : deux échelles d'analyse en région d'élevage. Thèse de Docteur-Ingénieur INA$\mathrm{PG}$ « Sciences Agronomiques », 152 pages + annexes. 
BENOîT M. (1990). — La gestion territoriale de l'activité agricole dans un village lorrain.

Mappemonde, 90/4 : 15-17.

BENoîT M. (1992). — Un indicateur des risques de pollution azotée nommé « BASCULE »

(Balance Azotée Spatialisée des systèmes de CULture de l'Exploitation. Fourrages, 129 : 95-110.

Benoît M., Deffontaines J.P., Gras F., Bienaimé E., Riela-Cosserat R. (1997). — Agriculture et qualité de l'eau. Une approche interdisciplinaire de la pollution par les nitrates d'un bassin d'alimentation. Cahiers Agriculture 1997 ; 6 : 97-105.

Benoît M., Deffontaines J.P., Hervé D., Lardon S., Le Ber F., Mullon C., PaPy F., Souchère V., Thinon P., TREUIL J.P. (1998). - Coordonner des choix de cultures sous contraintes

environnementales : des jeux de rôle aux modèles multi-agents. Colloque SMAGET Clermont-Ferrand, 5-8/10/98. $10 \mathrm{p}$.

BENOÎT M.,LE BER F., MARI J.F. (2001). - Recherche des successions de cultures et de leurs évolutions : analyse des données Ter-Uti en Lorraine.AGRESTE VISION Lorraine-Alsace. 31 : 23-30.

BENoîT M. \& PAPY F. (1997). - Pratiques agricoles et qualité de l'eau sur un territoire alimentant un captage. In : L'eau dans l'agro-écosystème, Riou C., Bonhomme R., Chassin P., Neveu A., Papy F. (eds), INRA Editions, 323-338.

BENOÎT M., SAINTÔT D., GAURY F. (1995). - Mesures en parcelles d'agriculteurs des pertes en nitrates. Variabilité sous divers systèmes de culture et modélisation de la qualité de l'eau d'un bassin d'alimentation. C.R. Acad. Agric., 81 (4) : 175-188.

BoulAine J. (1992). - Histoire de l'agronomie en France. Paris, Lavoisier Tec-Doc, 392 pages. Boulaine J. (1998). - D’Olivier de Serres à René Dumont. Paris, Lavoisier Tec-Doc, 220 pages.

BRUNET R. (1997). - Champs et contrechamps. Raisons de géographe. Belin, Paris, 319 p.

CANTER L.W. (1997). - Nitrates in groundwater. Lewis publisher. 263 pages.

CHRISTOPHe C., LARDon S., Monestiez P. (éd.) (1996). - Etude des phénomènes spatiaux en agriculture. INRA Editions, Paris, série colloque, 365 p.

CLAUDE C. (2001). - Dynamique des systèmes de culture dans le bassin de la Vesle : diagnostic et prospective. DEA de géographie Nancy II, INRA SAD Mirecourt. 40 p. + annexes.

DefFontAines J.P. (1998). - Les sentiers d'un géoagronome. Editions Arguments. 360 p.

Deffontaines J-P., Benoît M., Brossier J., Chia E., Gras F., Roux M. (Ed.) (1993). - Agriculture et qualité des eaux ; diagnostic et propositions pour un périmètre de protection. INRA-SAD, 334 pages.

De Koning G.H.J., Verburg Ph., VeldKamp A., Fresco L.O. (1999). - Multiscale modelling of land use change dynamics in Ecuador. Agricultural Systems, Vol. 61 : 77-93.

GAURY F. (1992). - Systèmes de culture et teneurs en nitrates des eaux souterraines. Dynamique passée et actuelle en région de polyculture-élevage sur le périmètre d'un gîte hydrominéral. Thèse de Doctorat de l'ENSA de Rennes, 229 pages + annexes

Gerber J., Holloway L., Seymour S., SteVen M., Watkins C. (1998). - New technologies and old knowledges : the impact of « precision farming »on the management of the English countryside. 
In : N. Croix(Ed.), Environnement et nature dans les campagnes, Nouvelles politiques, nouvelles pratiques. pp 187-204.

GIRARD C-M. \& BENOîT M. (1990). - Méthode de cartographie des prairies permanentes : application à la Lorraine sur les données SPOT. C. R. Acad. Sci. Paris, 310 (Série III) : 461-464. Gras R., Benoît M., Deffontaines J-P., Duru M., LAfArge M., LANGlet A., Osty P.-L. (1989). - Le fait technique en agronomie. Activité agricole, concepts et méthodes d'étude. Coéd. INRA-L'Harmattan, 160 pages.

GRIGG D.B. (1974). - The agricultural systems of the world. An evolutionary approach. Cambridge University Press. 358 p.

HEYdel L., BENoîT M., SCHIAVON M. (1997). — Estimation des apports de produits phytosanitaires à l'échelle de bassins d'alimentation. Agronomie (1997) 17, 25-33.

LE BER F. \& BENOÎT M. (1998). - Modelling the spatial organisation of land use in a farming territory. Example of a village in the «Plateau Lorrain ». Agronomie ; $18: 103-115$.

LEGAY J.M. (1986). - Méthodes et modèles dans l'étude des systèmes complexes. Cahiers de la Recherche-Développement, 11, 1-6.

LEGAY J.-M. (1997). - L'expérience et le modèle. Un discours sur la méthode. Paris, INRA (Sciences en questions), $111 \mathrm{p}$.

LeIGH R.A., Johnston A.E. (1994). - Long term Experiment in Agricultural and Ecological Sciences. Harpenden, UK, Rothamsted Experimental Station. CAB International, 428 p.

LE MOIGNE J.L. (1990). - La modélisation des systèmes complexes. Afcet systems. Dunod, paris, $178 \mathrm{p}$.

ManNion A.M. (1995). - Agriculture and Environnemental Change : Temporal ans Spatial Dimensions. Wiley. Chichester. 405 p.

MARTIN Ph., PAPY F., SOUChÈRE V., CAPILlON A. (1998). - Maîtrise du ruissellement et modélisation des pratiques de production ; Cahiers Agriculture, 7, 111-119.

MARY B., BEAUDOIN N., BENOÎT M. (1996). - Prévention de la pollution nitrique à l'échelle du bassin d'alimentation en eau. In : G. Lemaire et B. Nicolardot (eds), Maîtrise de l'azote dans les agrosystèmes, Reims. 19-20 octobre 1996, Les colloques nº 83, 289-312. INRA, Paris.

Maxime F., PAPy F., Mollet J.M.(1995). - Aide au raisonnement de l'assolement en grande culture. Cah. Agricultures, 4, 351-62.

Mignolet C., Benoît M., SAintôt D. (1997). - Systèmes d'élevage et risque de pollution azotée. Construction d'un indicateur de risque et application dans la plaine des Vosges. INRA Productions animales, 10 (4) : 275-285.

Mignolet C., ThÉnARD V., Benoît M., ANFrie M.N., Foissy D., Grosse M., TrommensChlaGer J.M.(1999). - Livestock farming systems and sustainable drinking water production : proposition of risk indicators at different organisational levels. Livestock Production Science, 61 : 307-313.

MoRLON P. \& BENoîT M. (1990). - Etude méthodologique d'un parcellaire d'exploitation agricole en tant que système. Agronomie, 1990 (6) : 499-508.

MuXART T., BLANDIN P., FRIEDBERG C. (1992). - Hétérogénéité du temps et de l'espace : niveaux d'organisation et échelles spatio-temporelles. In : Sciences de la nature, sciences de la société. M. Jollivet (ed.), Paris, France, CNRS, p. 403-425. 
OSTY P.L. (1994). - The farm enterprise and its environment. Proposals for structuring an appraisal of strategy. In : Brossier J., de Bonneval L. \& Landais E. (eds) Systems studies in agriculture and rural development, INRA (Science Update), 259-268.

OSTY P.L. (1996). - Methods and scales of intervention: what methodological renewal for System research? In : Systems-Oriented Research in Agriculture and Rural Development (Inter. Sympos., Montpellier, 21-25/11/94, Lectures and Debates, CIRAD, Montpellier, 169-172.

OSTY P.L., LARDON S., DE SAINTE-MARIE C. (1998). - Comment analyser les transformations de l'activité productive des agriculteurs : propositions à partir des systèmes techniques de production. Etudes et Recherches sur les Systèmes Agraires et Développement.

SAlou M.-C. (1992). - Elaboration de la qualité des eaux dans des bassins versants agricoles. Essai de modélisation en milieux calcaires (exemple des plateaux de Vicherey et d'Aboncourt), DEA Géographie, Metz ; INRA-SAD Mirecourt, 117 pages + annexes.

Sebillotte M. (1974). - Agronomie et Agriculture. Essai d'analyse des tâches de l'agronome.

Cahiers ORSTOM. Série Biologie. $\mathrm{N}^{\circ}$ 24, 3-25.

SEBiLlotTE M. (1996). - Systems Research and Action. Interdisciplinary Excursions. In : Sebillotte M. (dir.) Systems-Oriented Research in Agriculture and Rural Development.

Lectures and debates (International Symposium - Montpellier, Fr., 21-25 Nov. 1994). Montpellier, CIRAD, pp. 35-72.

THENAIL C. (1996). - Exploitations agricoles et territoire(s) : contribution à la structuration de la mosaïque paysagère. Thèse de l'Université Rennes I. 396 p.

VELDKAMP A., FRESCO L.O., 1997. - Exploring land use scenarios, an alternative approach based on actual land use. Agricultural Systems 55, 1-17.

VerbuRg P.H., De KONING G.H.J., KOK K., VelDKAMP A., BOUMA J., (1999). - A spatial explicit allocation procedure for modelling the pattern of land use change based upon actual land use. Ecological Modelling 116 (1), 45-61.

VEYRET Y. (dir.) 1998. - L'érosion entre nature et société, Paris, SEDES DIEM n² 22, 344 p.

ZANDER P., KÄCHELE H. (1999). - Modelling multiple objectives of land use for sustainable development. Agricultural Systems 59 (1999) 311-325.

\section{NOTES}

1. L'agriculture de précision se définit comme l'utilisation de techniques de spatialisation et contrôle des techniques agricoles permettant de différencier les modalités d'une opération technique au sein d'une même parcelle.

2. Département « Systèmes Agraires et Développement » de l'INRA.

3. Les données TerrUti informent sur l'utilisation du territoire français et son évolution. Relevée pour le SCEES par les DDAF, elles se présentent sous la forme de description d'utilisation de points répartis régulièrement (maillage systématique du territoire en environ 550000 points). Les premières informations remontant à 1982, nous pouvons construire des chroniques d'utilisation de chacun de ces points.

4. Les chaînes de Markov sont des traitements statistiques qui permettent de mettre en évidence les suites de données soit le long d'un axe temporel, ce que nous mobilisons, soit spatialement. Ces traitements mathématiques sont fréquemment utilisés pour retrouver les suites de gènes lors de séquençages génétiques. Ici nous rechercherons par ces méthodes les suites de cultures, ou 
pour être plus précis la probabilité que telle culture suive telle autre culture dans ce qui construit alors une succession culturale (Benoît, Le Ber, Mari, 2001).

5. Il existe une relation forte entre assolement et successions culturales où l'assolement est la spatialisation du choix par les agriculteurs de leurs couverts végétaux, les successions culturales étant la temporalisation de ces choix.

6. Part de la surface des exploitations qui contribue au bassin d'alimentation considéré.

\section{RÉSUMÉS}

L'activité agricole contribue à l'évolution de l'utilisation des territoires d'openfields. Nous proposons une démarche de diagnostic de l'évolution de l'utilisation de ces territoires qui articule les dimensions spatiales et fonctionnelles de l'activité agricole et intègre plusieurs niveaux d'organisation. Basé sur la notion d'assolement comme indicateur de l'organisation de l'espace agricole et comme révélateur des dynamiques territoriales, l'itinéraire méthodologique est illustré par deux exemples, celui de la protection des eaux dans des openfields lorrains sur bassins karstifiés et celui d'openfields du Bassin parisien dans un bassin versant tributaire de la Marne. La démarche vise à être reproductible dans d'autres situations et utilisable par les divers gestionnaires de ces territoires d'openfields.

Agricultural activity contributes to the development of the use of openfiel systems. We propose an approach for the analysis of the evolution of use of these areas, that articulates the spatial dimension and functions of agricultural activity and its several levels of organisation. The analysis is based on the idea of crop rotation, as indicated by the organisation of agricultural space and as revealed by territorial dynamics. The methodology is illustrated by two examples: the first concerning the protection of water in openfields on the karstic basins of Lorraine, the other concerning open-fields of the Paris Basin in a tributary catchment of the River Marne. The approach aims to be reproducible in the other situations and usable by the different administrators of these openfields.

Die Landwirtschaftliche Aktivität trägt zur Entwicklung der Nützung auf den offenen Feldfluren (openfields) bei. Wir regen eine Diagnose der Nützungsentwicklung dieser Ländereien an, welche die räumlichen und funktionalen Dimensionen der landwirtschaftlichen Aktivität ausdrückt und mehrere Organisationsniveaus integriert. Basierend auf dem Begriff der Fruchtfolge als Anzeiger der landwirtschaftlichen Raumorganisation und Dynamik wird die Methode durch zwei Beispiele illustriert - das Wasserschutzes in den Openfields Lothringens auf Kalkboden und das der Openfields des Pariser Beckens in einem der Marne tributären Becken. Die Untersuchung möchte auch in anderen Gebieten anwendbar sein und nutzbar durch di verschiedenen Formen dieser Openfieldgebiete. 
INDEX

Schlüsselwörter : Landschaftplannung, Landwirtschaftbetrieb, Wasserschutzgebiet

Keywords : agronomic diagnosic, catchment, crop rotation, mixed farming, Openfields, spatial analysis, underground water supply

Mots-clés : analyse spatiale, assolement, bassins d'alimentation, bassins versants, diagnostic agronomique, eaux souterraines, Openfields, polyculture-élevage

\section{AUTEURS}

\section{MARC BENOIT}

Institut National de la Recherche Agronomique ; station Systèmes Agraires et Développement 662, avenue Louis-Buffet - 88500 Mirecourt

\section{CÉLINE CLAUDE}

Institut National de la Recherche Agronomique ; station Systèmes Agraires et Développement 662, avenue Louis-Buffet - 88500 Mirecourt 